\title{
A DUAL APPROACH TO EMBEDDING THE COMPLEMENT OF TWO LINES IN A FINITE PROJECTIVE PLANE
}

\author{
LYNN MARGARET BATTEN
}

(Received 25 October 1989; revised 20 March 1990)

Communicated by Louis Caccetta

\begin{abstract}
Let $S$ be a finite linear space on $\nu \geq n^{2}-n$ points and $b=n^{2}+n+1-m$ lines, $m \geq 0, n \geq 1$, such that at most $m$ points are not on $n+1$ lines. If $m \geq 1$, except if $m=1$ and a unique point on $n$ lines is on no line with two points, then $S$ embeds uniquely in a projective plane of order $n$, or is one exceptional case if $n=4$. If $m \leq 1$ and if $\nu \geq n^{2}-2 \sqrt{n+3}+6$, the same conclusion holds, except possibly for the uniqueness.
\end{abstract}

1991 Mathematics subject classification (Amer. Math. Soc.) 05 B 05, 51 E 10.

\section{Introduction}

In [18], Totten proved that if $S$ is a linear space on $v=n^{2}-n$ or $v=$ $n^{2}-n+1$ points, $n \geq 2$, of which at least $n^{2}-n$ have degree $n+1$, and $b \leq n^{2}+n-1$ lines, then $S$ embeds in a finite projective plane of order $n$, with one exceptional case if $n=4$. The parameters are those of the complement of two lines in a finite projective plane of order $n$. He fixes $v$, the number of points, and allows the number of lines to vary. In this article, we take the dual approach of fixing $b$, the number of lines, and allowing the number of points to vary. The parameters are such that $m$ lines have been deleted from a projective plane, and in each case, all points but one have been deleted, but no point has two or more lines removed from it. In

This research was supported by NSERC grant A3485.

(C) 1991 Australian Mathematical Society 0263-6115/91 \$A2.00+0.00 
effect, since $v \geq n^{2}-n$, except for very small values of $n$, only one or two lines have been removed in this manner, perhaps with some additional points in a sporadic way; and we show that in almost all cases, $S$ re-embeds in a projective plane of order $n$.

Many articles in the literature have considered the problem of embedding a linear space $S$ with parameters $v, b$ and point and line sizes a function of an integer $n$, in a projective plane of order $n$. The particular situation of constant point size has been dealt with by Vanstone [19], McCarthy and Vanstone [9], Dow [6] and Beutelspacher and Metsch [5]. Constant line size implies constant point size in a linear space, so the dual approach to constant point size has been to allow the line sizes to range over a small number of values as in Batten and Totten [3], de Witte and Batten [20], Batten [1], Beutelspacher [4]. Another approach has been to fix the parameter $b$, while allowing $v$ to vary. The papers by Stinson [17], Erdös, Mullin, Sós and Stinson [7] and Metsch [10] figure here. A much broader approach of simply placing upper and lower bounds on $v$ and $b$ has been taken by de Witte [16] and Metsch [11].

As in Totten's paper[18], the motivation for the embedding problem has often come from examining the parameters of the complement of a configuration in a projective plane. For instance, Mullin and Vanstone [13], Mullin, Singhi and Vanstone [14], Ralston [15] and Montekhab [12] have each re-embedded the complement of a certain set of lines in a finite projective plane.

Finally, for a more comprehensive discussion of the embedding and complementation problems, we refer the reader to Batten and Beutelspacher [2].

\section{The setting}

A (finite) linear space is a (finite) set of $\nu$ elements called points together with a collection of $b$ sets of points called lines such that any two distinct points $p$ and $q$ belong to precisely one common line, denoted $p q$, and every line contains at least two points.

A projective plane of finite order $n$ is a finite linear space with $v=n^{2}+$ $n+1, n \geq 2$, in which each line has $n+1$ points and each point is on (in) $n+1$ lines. It is easy to see that in a projective plane any two lines meet in precisely one common point. For convenience, we shall call a triangle a projective plane of order 1.

A line with precisely $k$ points will frequently be referred to as a $k$-line, a point on precisely $k$ lines will frequently be referred to as a $k$-point.

In Sections 3 and 4, we prove the following result. 
Theorem. Let $S$ be a finite linear space on $\nu \geq n^{2}-n$ points and $b=$ $n^{2}+n+1-m$ lines, $m \geq 0, n \geq 1$, such that at most $m$ points are not on $n+1$ lines. Then if $m \geq 1$, except if $m=1$ and a unique point on $n$ lines is on no line with two points, then $S$ embeds uniquely in a projective plane of order $n$, with one exceptional case if $n=4$. If $m=0$ or $m=1$, and $\nu \geq n^{2}-2 \sqrt{n+3}+6, n \geq 1$, the same conclusion holds, except possibly for the uniqueness.

In order to prove the theorem, we shall make use of the following results.

THEOREM (Dow [6]). Let $S$ be a finite linear space on $b=n^{2}+n+1$ lines, $n \geq 1$, in which each point is an $(n+1)$-point. If $\nu \geq n^{2}-2 \sqrt{n+3}+6$, then $S$ can be embedded in a projective plane of order $n$.

THEOREM (Metsch [10]). Let $S$ be a finite linear space on $b=n^{2}+n+1$ lines, $n \geq 2$, in which each point is on at most $n+1$ lines. If $\nu>n^{2}-n / 6$, then $S$ can be embedded in a projective plane of order $n$, which is unique up to isomorphism.

\section{Proof of the theorem for $m \geq 2$}

Suppose some point $p$ is on $c<n$ lines. Counting $v$ at $p$ leads to $c \geq n-1$. Hence $p$ is on precisely $1 n$-line and $n-2(n+1)$-lines and $v=n^{2}-n$, or $p$ is on $n-1(n+1)$-lines and $v=n^{2}-n+1$. In the first case, the $n$-line $h$ on $p$ determines a spread of lines of $S$ (a set of pairwise disjoint lines such that each point of $S$ is on precisely one line). We introduce a new point $x$ corresponding to this spread, and say that $x$ is in each line of the spread. We get a linear space $S^{\prime}$ this way, in which each lines has $n-1, n$ or $n+1$ points, and $v=n^{2}-n+1$. In fact, we now have the second case. The main theorem of Batten [1] now indicates that $S^{\prime}$ is the complement of two lines less their point of intersection, in a projective plane of order $n, n \geq 1$.

We may therefore assume that each point of $S$ is an $n$ - or $(n+1)$-point.

CASE I. We suppose first of all that $(n+1)$-lines exist. (Clearly, no line can have more than $n+1$ points, and every line meets an $(n+1)$-line.)

If there is at most one $n$-point, then counting $b$ at an $(n+1)$-line leads to $b \geq n^{2}+n$, which is false. So there are at least two $n$-points, and a unique $(n+1)$-line $\ell$ on all $n$-points.

Counting $v$ using an $n$-point, we obtain $v \leq n+1+(n-1)^{2}=n^{2}-n+2$, which implies that each $n$-point is on at least $n-3 n$-lines. The case $n=1$ 
gives either a 2-point line or a triangle, each of which embeds in a projective plane of order $1 ; n=2$ produces a linear space on 4 points which embeds in the projective plane of order 2 . If $n=3$ and no $n$-point is on an $n$-line we obtain $v=6, b=10$ and $m=4$, a contradiction. Hence for $n \geq 3$, we may assume that each $n$-point is on at least one $n$-line.

Fix an $n$-point $p$ and an $n$-line $h$ on $p$. Any point not on $h$ is on at most one line missing $h$. Since all $n$-points are on $\ell, \ell \cap h=\{p\}$ and all lines meet $\ell$, it follows that $h$ determines a unique maximal partial spread of $n+1-(m-1)$ lines (that is, a maximal set of $n+1-m+1$ pairwise disjoint lines). We introduce a new point $x$ corresponding to this partial spread, and say $x$ is in every line of the partial spread. Fix a second $n$-point $q \neq p$ and an $n$-line $h^{\prime}$ on $q$. We introduce in the same way, a new point $y$ corresponding to the induced maximal partial spread.

The lines $h$ and $h^{\prime}$ meet in an $(n+1)$-point, and $(n-1) n+n+(n-2)=$ $n^{2}+n-2$ lines meet $h$ or $h^{\prime}$. Since $m \geq 2$, we have $n^{2}+n-2 \leq b \leq$ $n^{2}+n-1$.

(a) Suppose $b=n^{2}+n-1$, or equivalently, $m=2$. Then there is a unique line missing both $h$ and $h^{\prime}$. This line is common to both corresponding maximal partial spreads. So $x$ and $y$ belong to a common line of $S$.

For each of the $\geq n-3 n$-lines on $p$, we proceed in the manner described above to introduce new points $x_{1}, x_{2}, \ldots$. We also introduce a new line consisting of the point $q$ along with all the $x_{i}$. Similarly, for each of the $\geq n-3 n$-lines on $q$, we introduce new points $y_{i}$ and a new line consisting of $p$ and all the $y_{i}$. This new structure $S^{\prime}$ consisting of all points and lines of $S$ and all new points and lines is a linear space on $v^{\prime} \geq v+2 n-6 \geq n^{2}+n-6$ points, $b^{\prime}=n^{2}+n+1$ lines, and in which each point is on $n+1$ lines.

Either of the theorems cited in Section 2 can now be applied to give the embedding if $n \geq 6$. In particular, the theorem of Metsch also yields the fact that the embedding is unique. If $n=3$, counting points on the lines through a 3-point gives $v \geq 7$ and so $v^{\prime} \geq 9$. By Metsch, $S^{\prime}$ and therefore also $S$, embeds uniquely in a projective plane of order 3 . If $n=4$, using Metsch we can embed $S^{\prime}$, and also $S$, uniquely in a plane of order 4 if $v^{\prime} \geq 16$. Since $v \geq 12$, the only problematic cases are $v=12$ and $v=13$. If $v=12$ and $v^{\prime}=14$, and if $S^{\prime}$ contains no 4-lines, then each point of $S^{\prime}$ must be on precisely two 5-lines (by an easy computation). In this case, counting point-line incidences for 5-lines, we obtain the contradiction $14 \cdot 2$ is divisible by 5 . Hence $S^{\prime}$ contains a 4-line which can be used to produce a spread and hence a new point. We may therefore suppose, for $v=12$ or 13, that $S^{\prime}$ has 15 points, 21 lines and lines of maximum size 5. If 4-lines exist, introduce a new point and apply Metsch. If no 4-lines exist, an easy 
computation shows that each point of $S^{\prime}$ is on at least two 5-lines. If some point $x$ is on a 2-line $x y$, then $y$ is on at least three 5-lines which is not possible. Hence each point of $S^{\prime}$ is on precisely two 5-lines and three 3-lines. Letting

$$
\{1,2,3,4,5\},\{1,6,7,8,9\},\{1,10,11\},\{1,12,13\},\{1,14,15\}
$$

be the lines on the point 1 , it is not difficult to see that the following lines are determined:

$$
\begin{aligned}
& \{10,12,2,6,14\},\{10,13,15,7,3\},\{11,12,15,8,4\}, \\
& \{11,13,14,9,5\},\{10,8,5\},\{10,9,4\},\{11,7,2\},\{11,6,3\}, \\
& \{12,9,3\},\{12,7,5\},\{13,8,2\},\{13,6,4\}, \\
& \{14,8,3\},\{14,7,4\},\{15,9,2\},\{15,6,5\} .
\end{aligned}
$$

Since $S^{\prime}$ is unique and has the parameters of the complement of six points no three collinear in the projective plane of order 4 (a hyperoval), $S^{\prime}$, and therefore also $S$, embeds uniquely in the projective plane of order 4 .

Finally, if $n=5, v \geq 20$, and each 5-point must be on at least two 5lines. In this case, $v^{\prime} \geq 24$. If $v^{\prime} \geq 25$, Metsch gives a unique embedding. The only problematic case is therefore $v^{\prime}=24$. Once again, if 5-lines exist, we can introduce a new point and obtain an embedding. So suppose 5-lines do not exist. Then any point is on at least three 6-lines. If some point $x$ is on four or more 6-lines, then it must be on a 2-line $x y$, in which case $y$ is on at least five 6-lines, which is not possible. So every point is on three 6-lines, two 4-lines and one 3-line. Letting $x_{i}$ be the number of $i$-lines, counting point-line incidence yields $x_{3}=8, x_{4}=12, x_{6}=12$, while $b^{\prime}=31$ gives a contradiction.

(b) Suppose $b=n^{2}+n-2$, or equivalently, $m=3$. Then there is no line of $S$ missing both $h$ and $h^{\prime}$. The line $\ell$ contains a third $n$-point $r$.

We introduce a new system $S^{\prime}$ consisting of the points and lines of $S$ along with $x$ and $y$ and three new lines: $\{x, q\},\{y, p\},\{x, y, r\} . S^{\prime}$ is a linear space with $v^{\prime} \geq n^{2}-n+2$ points, $b^{\prime}=n^{2}+n+1$ lines, and each point on $n+1$ lines. If $n>4$, there is an $n$-line $h^{\prime \prime} \neq h$ on $p$. Moreover, $x \notin h^{\prime \prime}$ in $S^{\prime}$. However, the distinct lines $\{x, q\}$ and $\{x, y, r\}$ are both on $x$ missing $h^{\prime \prime}$, contradicting the fact that $x$ is on $n+1$ lines in $S^{\prime}$. Therefore $n \leq 4$.

If $n=3$ or 4 and a second $n$-line, $h^{\prime \prime}$, exists on $p$ as above, the same argument applies. If $n=3$ and $p$ is on a unique $n$-line, the only case to consider here is $v=7$ and $b=10$. The lines of $S$ can then be given by 
the sets

$$
\begin{aligned}
\{1,2,3,4\},\{1,5,6\},\{2,6,7\},\{3,5,7\},\{1,7\},\{2,5\}, \\
\\
\{3,6\},\{4,5\},\{4,6\},\{4.7\} .
\end{aligned}
$$

The embedding is given by the sets

$$
\begin{gathered}
\{1,2,3,4\},\{1,5,6,8\},\{1,7,12,13\},\{1,9,10,11\},\{2,5,11,13\}, \\
\{2,6,7,9\},\{2,8,10,12\},\{3,5,7,10\},\{3,6,11,12\}, \\
\{3,8,9,13\},\{4,5,9,12\},\{4,7,8,11\},\{4,6,10,13\} .
\end{gathered}
$$

This is a unique embedding in the projective plane of order 3.

Consider $n=4$. If $p$ is on no second $n$-line, then $v=12$. If a 5-point on $\ell$ is on an $n$-line, this introduces a partial spread of $S$ implying $v=11$ and a contradiction.

We prove now that there is a unique finite linear space $S$ with one 5-point line $\ell$, three 4-points, twelve points, eighteen lines, each 4-point on a unique 4-line, the 5-points on $\ell$ each on one 5-line, three 3-lines and one 2-line.

Let the points of $S$ be $1,2,3, \ldots, 12$, and the following sets be the lines on the 4-point 1:

$$
\{1,2,3,4,5\},\{1,6,7,8\},\{1,9,10\},\{1,11,12\} \text {. }
$$

Without loss of generality, the lines on the 4-point 2 are

$$
\{2,6,9,11\},\{2,7,12\},\{2,8,10\} \text {. }
$$

There are precisely three 4-lines, and they all meet each other. Two 4-lines pass through 6. (i) Suppose the third 4-line is not on 6. Then it is on either 7 or 8 . Without loss of generality, choose $\{3,7,10,11\}$ as a 4 -line on the third 4-point, 3. The point 11 is on two more lines, one a 3-line and one a 2 -line. Since the line on 8 and 11 must meet $\ell$, and since 4 and 5 play equivalent roles so far, we may choose $\{5,8,11\}$ and $\{4,11\}$ as lines. Now 3 and 6 must be on a line, and the only possibility is $\{3,6,12\}$. Thus $\{3,8,9\}$ is the remaining line on 3 . The 3 -lines on 4 are

$$
\{4,6,10\},\{4,7,9\},\{4,8,12\} \text {. }
$$

We need one 2-line and 2 3-lines now on 5. Thus either 5 and 6 are together on a 3-point line, or 5 and 7 are. This is not possible.

(ii) The third 4-line is therefore on 6 . It must be $\{3,6,10,12\}$. The other lines on 6 are $\{4,6\}$ and $\{5,6\}$. There must be two more 3-lines on 3 , and without loss of generality, these may be chosen to be $\{3,7,11\}$ and $\{3,8,9\}$. At this point, 4 and 5 still play interchangeable roles. We need three 3-lines on each of them, and so may choose these as

$$
\{4,7,9\},\{5,7,10\},\{4,8,12\},\{5,8,11\},\{4,10,11\},\{5,9,12\} \text {. }
$$


This gives us all eighteen lines of $S$. Hence $S$ is unique.

Now consider the projective plane $\pi$ of order 4 and let the points $p, q, r$, $x, y, z, s$ form a Fano configuration in $\pi$, such that the triples $p, q, r$ and $p, y, z$ and $q, x, y$ and $r, x, z$ and $s, r, y$ and $s, q, z$ and $s, p, x$ are collinear. In $\pi$ delete the lines $x y, x z$ and $y z$ and all their points except for the points $p, q$ and $r$. The complement of the deleted configuration in $\pi$ is a linear space with the parameters of the space we have just proved is unique, the $n$-lines in $\pi$ being $p s, q s$ and $r s$. Hence, the space $S$ embeds uniquely in the projective plane of order 4 .

CASE II. Suppose now that $(n+1)$-lines do not exist.

Suppose there is no $n$-point. Then counting $v$ at a point implies that $n$-lines exist. Let $\ell$ be such a line. It forms a spread of at least $v / n \geq n-1$ lines. But the facts that $n^{2}+1$ lines meet an $n$-line, and $b \leq n^{2}+n-1$ imply that there are precisely $n-1$ lines in the spread. These must all be $n$-lines, and so $v=n^{2}-n$ and $b=n^{2}+n-1$. Now any line not in the spread meets all lines of the spread, and thus is an $(n-1)$-line. Applying the theorem of Batten [1] we see that for $n \geq 4, S$ is the complement of two lines and all their points in a projective plane of order $n$, or, in case $n=4, S$ may be the exceptional case described in Totten [18] which does not embed in a projective plane of order 4 , but does embed in the projective plane of order 5 . In case $n=3$, it is easy to see that $S$ is once again the complement of two lines and all their points in the projective plane of order 3. For $n \leq 2, S$ does not exist.

Count $v$ using an $n$-point $p$. This gives $v \leq n^{2}-n+1$. So either every line on $p$ is an $n$-line, or there are $n-1 n$-lines on $p$ and a unique $(n-1)$-line.

Let $h$ be an $n$-line on the $n$-point $p$. Then $h$ gives rise to a maximal partial spread for which we introduce a new point $x$. Let $S^{\prime}$ be the new system consisting of all points and lines of $S$ where $x$ is said to be on any line of its partial spread, along with $x$ and all 2-point lines $\{x, q\}, q$ an $n$ point of $S$ not on $h$. Suppose $h$ contains $s n$-points. Then there are $m-s$ new 2-point lines in $S^{\prime}$. So $S^{\prime}$ has a total of $b^{\prime}=n^{2}+n+1-m+(m-s)=$ $n^{2}+n+1-s$ lines. Moreover, there are $s(n-1)+(n-s) n+1=n^{2}-s+1$ lines meeting $h$, including $h$ itself. So the maximal partial spread on $h$ contains $n^{2}+n+1-m-\left(n^{2}-s+1\right)+1=n-m+s+1$ lines. Thus in $S^{\prime}, x$ is on $n+1-m+s+(m-s)=n+1$ lines. Now $S^{\prime}$ has $v^{\prime}=v+1 \geq n^{2}-n+1$ points, and a unique $(n+1)$-line with $s \quad n$-points. All other points are $(n+1)$-points. If $s \geq 2$, we may apply case I to obtain the embedding.

If $s=1$, we take the remaining $n-1$ or $n-2 n$-lines of $S$ on $p$, which remain $n$-lines in $S^{\prime}$, and with each of these generate a spread and so 
introduce a new point. Let $x$ and $y$ be distinct new points generated from $n$-lines on $p$ in $S$, and let $h$ and $h^{\prime}$ be the corresponding $n$-lines. We claim that there is a unique line of $S^{\prime}$ on both $x$ and $y$. To see this, count lines of $S^{\prime}$ meeting $h$ or $h^{\prime}$. There are $(n-1) n+n+(n-1)=n^{2}+n-1$ such lines. Since $b^{\prime}=n^{2}+n$, we have the desired result.

Now the extended system $S^{*}$ obtained by adding these additional points to $S^{\prime}$ is a linear space with $v^{*} \geq n^{2}-1, b^{*}=n^{2}+n$, a unique $n$-point and all other points $(n+1)$-points. In fact, in $S^{*}$ either all lines on $p$ are $(n+1)$-lines, or $n-1$ lines on $p$ are $(n+1)$-lines and the $n$th line is an $(n-1)$-line. Let $q \neq p$ be any point, but choose it on the $(n-1)$-line on $p$ if that exists. Since all lines meet an $(n+1)$-line, $x$ is on $n n$-lines not on $p$. Each of these determines a maximal partial spread on $n$ lines. Adding $n$ new points appropriately and joining these in a single new line on $p$, we see that the resulting structure is a projective plane of order $n$ less 0,1 or 2 points. Hence we have a unique embedding.

\section{Proof of the theorem for $m=0$ or 1}

If $m=0$, each point is an $(n+1)$-point, and we apply Dow's theorem to obtain the desired result.

Suppose $m=1$. Each point is on $n$ or $n+1$ lines using the argument of Section 3. If there is no $n$-point, we obtain the contradiction $b \geq n^{2}+n+1$. Let $p$ be the unique $n$-point.

CASE I. Suppose that $(n+1)$-lines exist. Then $p$ is on all $(n+1)$-lines.

(i) Assume that $p$ is on a 2-line $\{p, x\}$. Let $\ell$ be an $(n+1)$-line, and $q \in \ell \backslash\{p\}$. If $q$ is on no $n$-line, then $v \leq n^{2}-n+1$. In this case, no point of $\ell \backslash\{p\}$ is on an $n$-line, and so any $n$-lines are on $p$. Now count $v$ using $x$. We get $v \leq n^{2}-2 n+2$. So $n^{2}-n \leq n^{2}-2 n+2$, or $n \leq 2$, which is impossible.

So each point of $\ell \backslash\{p\}$ is on at least one $n$-line. But every line on $p$ meets every $n$-line. So apart from $\{p, x\}, x$ is only on $n$-lines, and $v=n^{2}-n+2$.

For each $n$-line on $x$, we get a maximal partial spread on $n$-lines, and hence a new point. Join all new points to $p$ in a single line. The new structure $S^{\prime}$ is a linear space on $v^{\prime}=n^{2}+2$ points and $b^{\prime}=n^{2}+n+1$ lines. By Metsch, $S^{\prime}$, and hence also $S$, embeds uniquely in a finite projective plane of order $n$.

(ii) Assume that there are no 2-lines on $p$. If all lines on $p$ are $n$-or $(n+1)$-lines, then trivially, $S$ embeds in a unique way in a projective plane of order $n$. 
Let $h$ be a line with fewer than $n$ points on $p$. Let $x \in h \backslash\{p\}$. If $x$ is on no $n$-line, then $n^{2}-n-1 \geq v \geq n^{2}-2 \sqrt{n+3}+6$, a contradiction. Thus $x$ is on an $n$-line which produces a maximal partial spread which we use to introduce a new point. This new point is then joined to $p$ in a 2-point line yielding a linear space $S^{\prime}$ with $v^{\prime} \geq n^{2}-2 \sqrt{n+3}+7, b^{\prime}=n^{2}+n+1$, and each point an $(n+1)$-point. By Dow's theorem, $S^{\prime}$, and so also $S$, embeds in a projective plane of order $n$.

We note here, that for $v<n^{2}-2 \sqrt{n+3}+6$, such an embedding does not always exist. For example, a set of $t<n-1$ mutually orthogonal latin squares of order $n$ with no common orthogonal mate gives rise to a linear space $S^{\prime}$ with $b^{\prime}=n^{2}+n+1$ and each point an $(n+1)$-point, which cannot be embedded in a projective plane of order $n([6,8])$.

CASE II. Suppose that there are no $(n+1)$-lines. In this case counting $v$ on $p, n^{2}-n \leq v \leq n^{2}-n+1$.

If $v=n^{2}-n+1$, each line on $p$ is an $n$-line. Each gives rise via a spread to a new point, and so a new line on the $n$ new points. $S$ is easily seen to be a projective plane of order $n$ with one of its lines and all its points removed, and a second line with all its points but one removed.

If $v=n^{2}-n$, a single line on $p$ is an $(n-1)$-line and the other are $n$-lines. $S$ is as above, except that one more point on neither of the deleted lines, has been deleted.

\section{References}

[1] L. M. Batten, 'Linear spaces with line range $\{n-1, n, n+1\}$ and at most $n^{2}$ points', J. Austral. Math. Soc. Ser. A 30 (1980), 215-228.

[2] L. M. Batten and A. Beutelspacher, The Theory of Finite Linear Spaces, (Cambridge University Press, to appear).

[3] L. M. Batten and J. Totten, 'On a class of linear spaces with two consecutive line degrees', Ars Combin. 10 (1980), 107-114.

[4] A. Beutelspacher, 'Embedding linear spaces with two line degrees in finite projective planes', J. Geom. 26 (1986), 43-61.

[5] A. Beutelspacher and K. Metsch, 'Embedding finite linear spaces in projective planes II', Discrete Math. 66 (1987), 219-230.

[6] S. Dow, 'An improved bound for extending partial projective planes', Discrete Math. 45 (1983), 199-207.

[7] P. Erdös, R. C. Mullin, V. T. Sós and D. R. Stinson, 'Finite linear spaces and projective planes', Discrete Math. 47 (1983), 49-62.

[8] D. R. Hughes and F. C. Piper, Design Theory, (Cambridge University Press, Cambridge, New York, Melbourne, 1985).

[9] D. McCarthy and S. A. Vanstone, 'Embedding $(r, 1)$-designs in finite projective planes', Discrete Math. 19 (1977), 67-76. 
[10] K. Metsch, 'An improved bound for the embedding of linear spaces into projective planes', Geom. Dedicata 26 (1988), 333-340.

[11] _ _ 'An optimal bound for embedding linear spaces into projective planes', Discrete Math. 70 (1988), 53-70.

[12] M. S. Montekhab, Embedding of finite pseudo-complements of quadrilaterals, (Ph.D. thesis, University of London, 1985).

[13] R. C. Mullin and S. A. Vanstone, 'A generalization of a theorem of Totten', J. Austral. Math. Soc. Ser. A 22 (1976), 494-500.

[14] R. C. Mullin, N. M. Singhi and S. A. Vanstone, 'Embedding the affine complement of three intersection lines in a finite projective plane', J. Austral. Math. Soc. Ser. A 24 (1977), 458-464.

[15] T. Ralston, 'On the embeddability of the complement of a complete triangle in a finite projective plane', Ars Combin. 11 (1981), 271-274.

[16] D. R. Stinson, 'A short proof of a theorem of de Witte', Ars Combin. 14 (1982), 79-86.

[17] __ , 'The non-existence of certain finite linear spaces', Geom. Dedicata 13 (1983), 429-434.

[18] J. Totten, 'Embedding the complement of two lines in a finite projective plane', $J$. Austral. Math. Soc. Ser. A 22 (1976), 27-34.

[19] S. A. Vanstone, 'The extendibility of $(r, 1)$-designs', Proc 3rd Manitoba Conf. on Numerical Methods, Winnipeg (1973), 409-418.

[20] P. de Witte and L. M. Batten, 'Finite linear spaces with two consecutive line degrees', Geom. Dedicata 14 (1983), 225-235.

Department of Mathematics and Astronomy

University of Manitoba

Winnipeg, Manitoba

Canada R3T 2N2 